\title{
Nonpharmacologic Strategies and Topical Agents for Treating Atopic Dermatitis: An Update
}

\author{
Linda F. Stein Gold, MD,* and Lawrence F. Eichenfield, $\mathrm{MD}^{\dagger}$
}

\section{Abstract}

The signs and symptoms of atopic dermatitis can be safely and effectively controlled in most patients; in many cases, the disease can be improved to the point that signs and symptoms are absent or minimal. In addition, flares can be effectively controlled and, in some cases, prevented. New topical medications, improved strategies for the use of topical corticosteroids and topical calcineurin inhibitors, and judicious use of nonpharmacologic regimens-including bathing, bleach baths, and early use of emollients-have led to better disease management and improved quality of life for patients and their families.

Semin Cutan Med Surg 36(supp2):S42-S44

(C) 2017 published by Frontline Medical Communications

\section{Keywords}

Atopic dermatitis; bathing; bleach baths; calcineurin inhibitors, topical; corticosteroids, topical; eczema

A $\mathrm{s}$ the body of recent literature concerning atopic dermatitis (AD) continues to reshape consensus on many aspects of patient management, clinicians should not neglect to also update our messaging to our patients. It is important for patients and families to understand several key points. First, AD can be safely and effectively controlled in most patients. Second, with the availability of new medications and better strategies for the use of medications and nonpharmacologic regimens that have been the cornerstone of AD management for many years, it is now possible for treatment to improve AD to the point of minimal to no disease and symptoms. Third, flares can be effectively controlled and, in some cases, prevented.

\footnotetext{
*Director of Dermatology Research, Henry Ford Health System, Detroit, Michigan

Chief, Pediatric and Adolescent Dermatology, Professor of Dermatology and Pediatrics, Rady Children's Hospital, University of California, San Diego School of Medicine, San Diego, California

Publication of this CME/CE article was jointly provided by University of Louisville, Postgraduate Institute for Medicine, and Global Academy for Medical Education, LLC, and is supported by an educational grant from Anacor Pharmaceuticals, Inc. The authors have received an honorarium for their participation in this activity. They acknowledge the editorial assistance of Joanne Still, medical writer, and Global Academy for Medical Education in the development of this continuing medical education journal article.
}

Linda F. Stein Gold, MD, Consultant: Anacor. Grant/Research: Anacor, GlaxoSmithKline. Data Monitoring Committee: Otsuka America Pharmaceutical, Inc.

Lawrence F. Eichenfield, MD, Advisory Board/Speaker: Valeant Pharmaceuticals North America LLC. Consultant: Anacor/Pfizer Inc., Eli Lilly and Company, Genentech, Inc., Otsuka/Medimetriks

Pharmaceuticals, Inc., Sanofi Genzyme/Regeneron Pharmaceuticals,

TopMD, Valeant. Investigator: Sanofi Genzyme/Regeneron.

Address reprint requests to: Linda F. Stein Gold, MD, 2360 Heronwood Drive, Bloomfield Hills, MI 48302; lstein1@hfhs.org
Patients and caregivers should know that lack of full control of signs and symptoms of AD need not and should not be simply tolerated, and visits to the clinician should not be put off until a flare occurs or until signs and symptoms progress to an unacceptable threshold of severity. Strong evidence from prospective studies, reviewed and evaluated by a panel of experts, demonstrates that some treatments for $\mathrm{AD}$ that have been in use for many years should still be considered valuable components of a comprehensive treatment regimen (Table).

\section{Bathing Frequency/Duration}

Clinicians differ on their recommendations for the frequency of bathing and duration of immersion time. Unfortunately, no good evidence is available to establish firm guidelines. Patients and caregivers who avoid daily bathing because of the perception that frequent bathing further dries already xerotic skin should understand that water does not contribute to skin dryness. Frequent — even daily or twice-daily — baths are helpful in hydrating the skin, provided that a liquid nonsoap cleanser is used that is appropriate for patients with $\mathrm{AD}$ and that moisturization is always included in the bathing routine - a regimen referred to as "soak and seal." In addition, frequent bathing is especially helpful and important for patients with exudative eczema and may decrease bacterial colonization of the skin.

When inflammation is severe, applications of towels soaked in warm to hot (but not scalding) water over a topical corticosteroid agent or moisturizer can soothe discomfort and calm erythema. Application of plastic food wrap over the towels prolongs the time between resoaking and reapplication.

Experience shows that short baths, lasting 5 to 10 minutes, are best. In addition to hydrating and cleansing the skin, bathing improves penetration of topical medications and aids in debridement of infected and necrotic tissue. A moisturizer and/or emollient ointment or cream (rather than lotion) should be applied promptly after immersion, within a few minutes and while the skin is still moist.

\section{Infection Control}

Because the skin barrier is defective in patients with AD, patients have an increased susceptibility to infections, including viral (especially molluscum contagiosum and herpes simplex virus), fungal (tinea and Malassezia), and bacterial (Staphylococcus aureus).

$S$ aureus colonization is a common problem and poses an ongoing risk for secondary bacterial infection in patients with $\mathrm{AD}$, and numerous investigators have explored strategies to manage colonization and prevent infection. In one study, Huang and colleagues ${ }^{1}$ demonstrated that treatment of $S$ aureus colonization can decrease the severity of AD. In that 3-month, randomized, investigator-blinded, placebocontrolled study, 31 patients between 6 months and 17 years of age with moderate to severe $\mathrm{AD}$ and secondary bacterial

(C) 2017 Frontline Medical Communications 1085-5629/13/\$-see front matter doi:10.12788/j.sder.2017.011 
infections received a 14-day course of oral cephalexin plus either intranasal mupirocin ointment ( 5 consecutive days per month) and a twice-weekly bleach bath or intranasal petrolatum ointment and plain water baths. The researchers found that the incorporation of bleach baths and intranasal mupirocin resulted in a statistically significant improvement in the Eczema Area and Severity Index than did the placebo regimen.

More recently, Shi and colleagues ${ }^{2}$ addressed the value of bleach baths - the addition of 4 ounces ( $1 / 2$ cup) of household bleach to a standard-sized bathtub of water-has both antiinflammatory and anti-infective properties, and can be helpful in both preventing infection and decreasing inflammation in susceptible patients.

Although no standard has been set for the frequency of bleach baths, it seems prudent to recommend that patients with severe AD and recurrent infections use a bleach bath at least three times weekly; consider daily bleach baths for patients with very severe AD and very frequent infection recurrences. ${ }^{3}$ Patients who prefer to shower rather than bathe can achieve the same benefit by using a "bleach spray": a mixture of 1 teaspoon of household bleach in 64 ounces (a half gallon) of water, applied to the skin during the shower. Commercial sodium hypochlorite sprays are sold over-thecounter, although the mixture described is easy to prepare and is considerably less expensive.

\section{TABLE Recommended Nonpharmacologic Interventions for Atopic Dermatitis*}

\author{
Moisturizers \\ Integral in atopic dermatitis (AD) \\ Strong evidence that moisturizer use can reduce disease \\ severity and need for pharmacologic treatment \\ Should be applied soon after bathing to improve \\ skin hydration

\section{Bathing} \\ Suggested as part of treatment and maintenance (no \\ standard established for frequency or duration) \\ Addition of oils, emollients, and most other additives to bath \\ water and use of acidic spring water are not recommended \\ at this time (evidence insufficient)
}

\section{Cleansers}

Limited use of nonsoap cleansers that are neutral or low $\mathrm{pH}$, hypoallergenic, and fragrance-free

\section{Wet-wrap treatments}

Use with or without topical corticosteroids

Recommended for patients with moderate to severe AD to decrease disease severity and transepidermal water loss during flares

*A summary of evidence-based recommendations from the American Academy of Dermatology Guidelines of Care for the Management of Atopic Dermatitis.

Source: From Eichenfield LF, Tom WL, Berger, et al. Guidelines of care for the management of atopic dermatitis: Section 2. Management and treatment of atopic dermatitis with topical therapies. J Am Acad Dermatol. $2014 ; 71: 116-132.8$

\section{Topical Corticosteroid Therapy Maintenance}

The use of topical corticosteroid therapy to bring an acute flare of AD under control is an undisputed strategy. However, the duration of topical corticosteroid treatment is a topic of ongoing debate, with some clinicians advocating cessation of treatment once the skin is clear and others promoting the benefits of long-term maintenance therapy.

Hanifin and colleagues ${ }^{4}$ examined the question of topical corticosteroid maintenance in a study of 372 children and adults with moderate to severe AD. All patients were treated with fluticasone propionate cream $0.05 \%$, twice daily for 4 weeks, plus emollients. After 4 weeks, patients were assigned to either an active-treatment group, continuing fluticasone applications on a maintenance schedule of twice weekly, or to a vehicle-only group. Hanifin et al reported that at week 20 , the patients in the vehicle-only group were 7 to 8 times more likely to experience a relapse than were the patients who continued maintenance treatment with the corticosteroid.

\section{Topical Calcineurin Inhibitors}

The two currently available topical calcineurin inhibitors (TCIs), pimecrolimus and tacrolimus, have a long history of safety and efficacy in patients with AD. Unlike topical corticosteroids, these agents can be used on any affected body surface area (BSA), including the face. The efficacy and safety of these agents have been demonstrated in clinical trials.

Eichenfield and colleagues ${ }^{5}$ studied the efficacy and safety of pimecrolimus cream $1 \%$ vs vehicle in two 6-week, randomized, multicenter studies involving 403 children and adolescents with mild (30\%), moderate (60\%), and severe $(9 \%)$ AD. All patients had BSA involvement of at least $5 \%$. As early as day 8 , a statistically significant improvement was seen on the investigator's global score of clear or almost clear in the active-treatment group vs vehicle $(P \leq 0.05)$.

In a 12-week randomized, double-blind, vehicle-controlled study, Paller and colleagues ${ }^{6}$ evaluated the safety and efficacy of tacrolimus ointment $0.03 \%$ and $0.1 \%$ in pediatric patients with moderate to severe AD. A total of 352 children between 2 and 15 years of age (mean age, 6.1 years) were enrolled; $61.5 \%$ had severe AD at baseline; $83.5 \%$ of patients had involvement of the head/face and/or neck. At week 12 , significantly more patients in the tacrolimus treatment groups had clinical improvement of at least $90 \%$ than did patients in the vehicle group $(P \leq 0.001)$. The authors noted that in the active-treatment groups, improvements in AD signs and symptoms, BSA affected, and patients' assessment of pruritus were seen early in the study and were maintained through week 12. Several adverse events occurred more frequently in the higher-concentration tacrolimus treatment groups than in the vehicle group: a sensation of burning, pruritus, varicella, and blisters (the incidence of varicella and blisters was $<5 \%$ ). (Note that only the $0.03 \%$ concentration is approved by the US Food and Drug Administration for use in patients $<15$ years of age.)

Gollnick and colleagues ${ }^{7}$ studied pimecrolimus cream $1 \%$ in the long-term management of AD in adults, with the goal of determining the effectiveness of this agent in preventing the progression of flares. The 543 enrolled patients were at 
least 18 years of age and had mild or moderate AD. Their $\mathrm{AD}$ was clear or almost clear before they were randomized to receive pimecrolimus cream $(n=277)$ or vehicle only $(n=266)$ in this 26-week study. Twice-daily applications of the study medication (either pimecrolimus or vehicle) were started as soon as a patient had any sign or symptom of a relapse. If the disease worsened, patients were permitted to add a moderately potent topical corticosteroid after the study medication had been used for at least 3 days. The primary endpoint was the number of days elapsed before corticosteroid treatment was needed to control a flare. The investigators reported that the mean number of corticosteroid-free days was significantly higher in the active-treatment group (152 days) than in the vehicle group (138.7 days) $(P<0.001)$; moreover, the mean number of flares requiring corticosteroid treatment was lower in the pimecrolimus group $(P=0.0014)$.

The guidelines for topical treatment of $\mathrm{AD}^{8}$ note that TCIs are valuable as corticosteroid-sparing agents; TCIs should be prescribed for the treatment of actively affected areas. If necessary, a topical corticosteroid can be used with a TCI to bring an AD flare under control.

The guidelines also recommend use of these agents, proactively, for relapse prevention. The use of TCIs as maintenance therapy should be intermittent, with applications 2 or 3 times weekly on areas that commonly flare. Such use is further supported by the results of a study by Luger and colleagues, ${ }^{9}$ who reviewed the use of TCIs in 21 trials including 5,825 pediatric patients with $\mathrm{AD}$. The authors described the safety data as "well-reported": Less than 5\% of patients reported discontinuation of TCI treatment because of adverse events; cutaneous and systemic adverse events were similar in the TCI and vehicle groups; and no cases of lymphoma were reported.

\section{Conclusion}

Effective control of atopic dermatitis involves a comprehensive approach utilizing good skin care principles as well as nonpharmacologic and pharmacologic treatments. Proactive management and maintenance therapy can prolong remission and provide significant relief.

\section{References}

1. Huang JT, Abrams M, Tlougan B, Rademaker A, Paller AS. Treatment of Staphylococcus aureus colonization in atopic dermatitis decreases disease severity. Pediatrics. 2009;123:e808-e814.

2. Shi VY, Foolad N, Omelas JN, et al. Comparing the effect of bleach and water baths on skin barrier function in atopic dermatitis: A split-body randomized controlled trial. Br J Dermatol. 2016;175:212-214.

3. Krakowski AC, Eichenfield LF, Dohil MA. Management of atopic dermatitis in the pediatric population. Pediatrics. 2008;122:812-824

4. Hanifin J, Gupta AK, Rajagopalan R. Intermittent dosing of fluticasone propionate cream for reducing the risk of relapse in atopic dermatitis patients. $\mathrm{Br} J$ Dermatol. 2002; 147:528-537.

5. Eichenfield LF, Lucky AW, Boguniewicz M, et al. Safety and efficacy of pimecrolimus (ASM 981) cream 1\% in the treatment of mild and moderate atopic dermatitis in children and adolescents. J Am Acad Dermatol. 2002;46:495-504.

6. Paller A, Eichenfield LF, Leung DY, Stewart D, Appell M. A 12-week study of tacrolimus ointment for the treatment of atopic dermatitis in pediatric patients. $J$ Am Acad Dermatol. 2001;44(suppl):S47-S57.

7. Gollnick H, Kaufmann R, Stough D, et al. Pimecrolimus cream $1 \%$ in the longterm management of adult atopic dermatitis: Prevention of flare progression. A randomized controlled trial. Br J Dermatol. 2008;158:1083-1093.

8. Eichenfield LF, Tom WL, Berger, et al. Guidelines of care for the management of atopic dermatitis: Section 2. Management and treatment of atopic dermatitis with topical therapies. J Am Acad Dermatol. 2014;71:116-132.

9. Luger T, Boguniewicz M, Carr W, et al. Pimecrolimus in atopic dermatitis: Consensus on safety and the need to allow use in infants. Pediatr Allergy Immunol. 2015;26:306-315. 